\title{
Decomposition of the Kostlan-Shub-Smale model for random polynomials
}

\author{
V. Gichev
}

\begin{abstract}
Let $\mathcal{P}_{n}$ be the space of homogeneous polynomials of degree $n$ on $\mathbb{R}^{m+1}$. We consider the asymptotic behavior of some coefficients relating to the decomposition of $\mathcal{P}_{n}$ into the sum of $\mathrm{SO}(m+1)$-irreducible components. Using the results, we prove that a random Kostlan-Shub-Smale polynomial $u \in \mathcal{P}_{n}$ can be approximated by polynomials of lower degree in the Sobolev spaces $H^{k}\left(S^{m}\right)$ on the unit sphere $S^{m}$ with small error and probability close to 1 . For example, if $l_{n}>\sqrt{(m+2 k+8 \varepsilon) n \ln n}$, then the inequality $\operatorname{dist}\left(u, \mathcal{P}_{l_{n}}\right)<A n^{-\varepsilon}\|u\|$ holds for any sufficiently large $n$ with probability greater than $1-B n^{-2 \varepsilon}$, where dist and \|\| are the distance and norm in $H^{k}\left(S^{m}\right)$, respectively, $\varepsilon \in(0,1)$, and $A, B$ depend only on $m$ and $k$. If $l_{n}>\varepsilon n$, then both the approximation error and the deviation of probability from 1 decay exponentially.
\end{abstract}

\section{Introduction}

Let $G$ be a compact Lie group acting on a Riemannian manifold $M$ by isometries, $\mathcal{E}$ be a finite dimensional $G$-invariant subspace of $C^{\infty}(M)$. For $u \in \mathcal{E}$, set $N_{u}=u^{-1}(0)$. Let $\sigma$ be a $G$-invariant probability measure on $\mathcal{E}$. Then the Hausdorff measure $\mathfrak{h}^{m-1}\left(N_{u}\right)$ of $N_{u}$, where $m=\operatorname{dim} M$, is a random variable whose distribution depends on $\sigma$. There are other metric quantities which can be considered in this setting, for example, the Euler characteristic of $N_{u}$ (see [16], [5]). Most of the known results were proved for the Gaussian distributions in $\mathcal{E}$ and the uniform distribution in the unit sphere in $\mathcal{E}$.

We consider the case $G=\mathrm{SO}(m+1), M=S^{m}=\mathrm{SO}(m+1) / \mathrm{SO}(m)$, the unit sphere in $\mathbb{R}^{m+1}$, and $\mathcal{E}=\mathcal{P}_{n}$, the space of real homogeneous of degree $n$ polynomials on $\mathbb{R}^{m+1}$. Let $\mathcal{H}_{j}$ be the subspace of all harmonic polynomials in $\mathcal{P}_{j}$. There is the well known $\mathrm{SO}(m+1)$-invariant decomposition

$$
\begin{array}{r}
\mathcal{P}_{n}=\sum_{j \in J_{n}}|x|^{n-j} \mathcal{H}_{j}, \\
J_{n}=\{j \in \mathbb{Z}: 0 \leq j \leq n, n-j \text { even }\} .
\end{array}
$$

The restriction onto $S^{m}$ is an injective map on the space $\mathcal{P}_{n}$. The space $\sum_{j \in J_{n}} \mathcal{H}_{j}$ has the same property since it consists of harmonic polynomials. By (1.1), it has

2010 Mathematics Subject Classification. Primary 60H25, 60G60; Secondary 43 A85.

The author was partially supported by the grant of the Norwegian Research Council \#204726/V30. 
the same traces on $S^{m}$. We denote by $\mathcal{P}_{n}, \mathcal{H}_{j}$ the function spaces on $S^{m}$ as well as on $\mathbb{R}^{m+1}$ hoping that no confusion will occur. Due to this convention,

$$
\mathcal{P}_{j} \subseteq \mathcal{P}_{n} \text { if } j \in J_{n} .
$$

The investigation of random polynomials was initiated by papers 3 by Bloch and Polya, and [14, 15 by Littlewood and Offord. In [11, M. Kac proved an exact integral formula for the expectation of the number of real zeroes of random polynomials of one variable with standard Gaussian coefficients (i.e., having expectation 0 and variance 1). Kostlan (see 12, 6) found a geometric proof of this formula. For any $a \in \mathbb{R}^{n+1}$, common points of the hyperplane $a^{\perp}$ and the moment curve $\gamma(x)=\left(1, x, \ldots, x^{n}\right)$ are in one-to-one correspondence with zeroes of the polynomial $\langle a, \gamma(x)\rangle$. The same is true for the central projection $\tilde{\gamma}(x)=\frac{\gamma(x)}{|\gamma(x)|}$ of $\gamma$ onto the unit sphere $S^{n}$. Set $f(a)=\operatorname{card}\left(a^{\perp} \cap \tilde{\gamma}\right)$. Since $f(a)$ is homogeneous of degree 0 , we can compute the expectation of the number of zeroes integrating $f$ over $S^{n}$; on the other hand, the integral is proportional to the length of $\tilde{\gamma}$ due to a Crofton type formula.

This method can be extended to the other function spaces and inner products. Kostlan noted that the distribution on the space of polynomials whose coefficients are independent Gaussian with the variance $\left(\begin{array}{l}n \\ j\end{array}\right)$ at $x^{j}$ has a hidden symmetry: it can be lifted onto the space of homogeneous degree $n$ polynomials of two variables as an $\mathrm{SO}(3)$-invariant distribution. In [12, Kostlan found the expectation of the volume of solutions to a random system of equations $u_{j}(x)=0$, where $u_{j} \in \mathcal{P}_{n}$ are independent random polynomials, $j=1, \ldots, k$, where $k \leq m$. It is proportional to $n^{\frac{k}{2}}$. If $k=m$, then it is the mean number of solutions and is equal to $n^{\frac{m}{2}}$. Shub and Smale in the paper [17. extended this result onto the case of different degrees: the expectation of the number of solutions to the system $u_{1}(x)=\cdots=u_{m}(x)=0$, $u_{j} \in \mathcal{P}_{n_{j}}$, is equal to $\sqrt{n_{1} \ldots n_{m}}$.

In the paper [16, Podkorytov introduced a parameter of a Gaussian $\mathrm{SO}(m)$ invariant distribution in $\mathcal{P}_{n}$ and found an explicit formula for the expectation of the Euler characteristic of $N_{u}$ which is a function of this parameter. In higher codimensions (i.e., for the varieties $N_{u_{1}} \cap \ldots \cap N_{u_{k}}$, where $k \leq m$ ), Bürgisser computed the expectation of the Euler characteristic in the paper [5]. His proof involves Weyl's tube formula.

The space $M=G / H$ is called isotropy irreducible if $H$ is irreducible in $T_{o} M$, where $o$ is the base point of $M$ corresponding to $H$. Such a space admits the unique up to a scaling factor invariant Riemannian metric. Hence any equivariant nonconstant mapping of $M$ into a Riemannian $G$-manifold is a finite covering and a local metric homothety onto its image. We assume $M$ isotropy irreducible in what follows.

The $G$-invariant inner product in $\mathcal{E}$ defines two parameters relating to the geometry of the evaluation mapping ev $\mathcal{E}_{\mathcal{E}}: M \rightarrow \mathcal{E}$ (see Section 2 for precise definitions). We denote them by $c$ and $s$ throughout the paper. The mapping ev is an immersion of $M$ into the sphere of radius $c$ :

$$
c=\left|\mathrm{ev}_{\mathcal{E}}(p)\right|, p \in M .
$$


Thus, $\iota=\frac{1}{c} \mathrm{ev}_{\mathcal{E}}$ is a non-constant mapping into the unit sphere $\mathcal{S}$ in $\mathcal{E}$. Let $s$ denote the coefficient of local metric homothety for $\iota$ :

$$
s=\frac{\left|d_{p} \iota(v)\right|_{\mathcal{E}}}{|v|_{T_{p} M}},
$$

This parameter was introduced in 9 . For Gaussian distributions, $s^{2}$ is the Podkorytov parameter.

The parameters $c$ and $s$ are essential ingredients in the formulas for the averages and fluctuations of some geometric quantities. Here is an example. Locally, the mapping $\iota$ multiplies the $k$-dimensional Hausdorff measure $\mathfrak{h}^{k}$ on $s^{k}$. This makes it possible to compute or estimate Hausdorff measure of a set $X$ in $M$ applying Federer's kinematic formula for spheres ([7, Theorem 3.2.48]) to $\iota(X)$. Let $X=N_{u}$, $u \in \mathcal{S}$. Integrating over $\mathcal{S}$ we get $\mathrm{E}\left(\mathfrak{h}^{m-1}\left(N_{u}\right)\right)=\frac{\varpi_{m-1}}{\varpi_{m}} s$, where $\varpi$ and $\varpi_{k}$ are volumes of $M$ and the unit sphere $S^{k}$ in $\mathbb{R}^{k+1}$, respectively. For the expectation of measures of the intersections of the sets $N_{u}$, there is a similar expression with the product of the coefficients of metric homothety.

Sometimes, it is possible to find $s$ following the definition (i.e., applying (1.4)). This is the case in the Kostlan-Shub-Smale model (see Section 3 for the definition). If $\mathcal{E}$ is an eigenspace of the Laplace-Beltrami operator $\Delta_{M}$ and $\lambda$ is the eigenvalue of $-\Delta_{M}$, then

$$
s=\sqrt{\frac{\lambda}{m}}
$$

independently of the choice of the invariant inner product. Since $M$ is isotropy irreducible, any $G$-irreducible component of $\mathcal{E}$ is an eigenspace of $\Delta_{M}$. In general, $s$ depends on the irreducible components of $\mathcal{E}$ and the inner product in $\mathcal{E}$. For the norm of $L^{2}(M)$ in $\mathcal{E}$ the answer is given in [10, Lemma 1]: if

$$
\mathcal{E}=\mathcal{E}_{1} \oplus \cdots \oplus \mathcal{E}_{l}
$$

where the summands are $G$-irreducible eigenspaces of $\Delta_{M}$, then

$$
s^{2}=\nu_{1} s_{1}^{2}+\cdots+\nu_{l} s_{l}^{2},
$$

where $s_{j}$ is the coefficient of metric homothety for $\mathcal{E}_{j}$, which is subject to (1.5), and $\nu_{j}=\frac{\operatorname{dim} \mathcal{E}_{j}}{\operatorname{dim} \mathcal{E}}, j=1, \ldots, l$. In Section 2 we show that (1.7) holds for any invariant inner product in $\mathcal{E}$ and $\nu_{j}=\frac{c_{j}^{2}}{c^{2}}$, where $c_{j}$ and $c$ are the parameters for $\mathcal{E}_{j}$ and $\mathcal{E}$, respectively. The coefficients $\nu_{j}$ depend on the choice of the inner product but $s_{j}$ are always subject to (1.5). This makes it is possible to find $c$ and $s$ for the rescaling inner products in $\mathcal{E}$. Section 2 contains the preparatory material which can be used if $\mathcal{E}$ is a finite dimensional $G$-invariant function space with arbitrary spectrum on any isotropy irreducible homogeneous space $M$.

In the case $M=S^{m}, \mathcal{E}=\mathcal{P}_{n}$, and the decomposition (1.1), $s \sim \frac{n}{\sqrt{m+2}}$ as $n \rightarrow \infty$ for the $L^{2}\left(S^{m}\right)$-norm in $\mathcal{E}$. In the Kostlan-Shub-Smale model, $s=\sqrt{n}$ independently of $m$. In Proposition 3.1 we collect the formulas for the parameters $c, s$ in the spaces $\mathcal{P}_{n}$ and $\mathcal{H}_{j}$, the coefficients $\nu_{j}$ and the rescaling factors for the $L^{2}\left(S^{m}\right)$ and Kostlan-Shub-Smale ensembles. In the recent paper [8], Fyodorov, Lerario, and Lundberg found, among other results, these factors and their scaling limit as $n \rightarrow \infty$ which is equal to $e^{-\frac{x^{2}}{4}}$ up to a normalizing factor. 
The coefficients $\nu_{j}$ have a peak near $\sqrt{(m-1) n}$ and decay very fast when $j$ grows. The peak can be localized in an interval of length $\frac{m+5}{2}$ and is sharpening as $m$ grows. In Theorem 4.2, we compute the scaling limit of $\nu_{j}$. Up to a normalizing factor, it is equal to $\left(t^{2} e^{1-t^{2}}\right)^{\frac{m-1}{2}}$.

The main result of the paper is Theorem 5.3. It implies that a random KostlanShub-Smale polynomial of degree $n$ admits a good approximation in the Sobolev spaces by polynomials of degree less than $\sqrt{(m+2 k+\varepsilon) n \ln n}$ with high probability. For example, if $k=0, n$ is sufficiently large,

$$
n>l_{n}>2 \sqrt{m n \ln n}
$$

and $n-l_{n}$ is even, then the inequality

$$
\operatorname{dist}\left(u, \mathcal{P}_{l_{n}}\right)<\varepsilon_{n}|u|
$$

holds with the probability greater than $1-\eta_{n}$, where

$$
\varepsilon_{n}=a n^{-\frac{m}{2}}, \eta_{n}=b n^{-\frac{m}{2}},
$$

dist stands for the distance in $L^{2}\left(S^{m}\right)$, and $a, b$ are independent of $n$. If $l_{n}>$ $\alpha n$, where $0<\alpha<1$, then $\varepsilon_{n}$ and $\eta_{n}$ may both decay exponentially when $n$ grows. Theorem 5.3 provides . This is not clear yet if $\sqrt{(m+2 k) n \ln n}$ is actually the critical rate of growth for the approximation in $H^{k}\left(S^{m}\right)$ by polynomials of lower degree. Since $\operatorname{dim} \mathcal{P}_{n}$ grows as $n^{m}$ as $n \rightarrow \infty$, the measure concentration phenomenon works in this situation. Hence the inequality above holds with high probability due to the fast decay of the coefficients $\nu_{j}$.

Throughout the paper, we fix $m$ and drop it in the notation assuming

$$
1<m<n .
$$

We use the notation || for the Euclidean norms and $\langle$,$\rangle for the corresponding$ inner product. The base point $o$ of $M=G / H$ is the class $H$. If $M$ is the unit sphere $S^{m}$ in $\mathbb{R}^{m+1}$, then $o=(1,0, \ldots, 0)$. In the notation $L^{2}(M)$, the invariant probability measure on $M$ is assumed. Also, $d u$, $d x$, etc. stands either for the Lebesgue measure in an Euclidean space or for the invariant probability measure on a compact homogeneous space (in particular, on $S^{m}$ ).

Acknowledgements. I am grateful to the unknown referee for useful comments and for making me aware of the papers [8] and [13. A part of this work was done during my stay at the University of Bergen, in the warm and friendly atmosphere created by Irina Markina and Aleksandr (Sasha) Vasiliev. Sasha's untimely decease is an irreplaceable loss, he was a wonderful person and a gifted mathematician. This paper is dedicated to his memory.

\section{The coefficients corresponding to invariant Euclidean structures}

Since $M$ is isotropy irreducible, the invariant Riemannian metric on it is unique up to a scaling factor. Hence it is the quotient of some bi-invariant metric on $G$. This implies that any $G$-invariant finite dimensional function space on $M$ is $\Delta_{M^{-}}$ invariant (the introduction in [10] contains more details; for $M=S^{m}$ this is true because the summands in 1.1 are irreducible and pairwise non-equivalent). Hence the summands of the $G$-invariant orthogonal decomposition (1.6) are eigenspaces of $\Delta_{M}$. Let $\lambda_{j}$ denote the eigenvalue of $-\Delta_{M}$ on $\mathcal{E}_{j}$. We assume $\mathcal{E}_{j} \neq 0$ for all $j=1, \ldots, l$. 
The evaluation mapping ev $: M \rightarrow \mathcal{E}$ is defined by the identity

$$
\left\langle\operatorname{ev}_{\mathcal{E}}(p), u\right\rangle=u(p),
$$

where $p \in M, u \in \mathcal{E}$. Then $\iota(p)=\frac{\mathrm{ev}_{\mathcal{E}}(p)}{\left|\mathrm{ev}_{\mathcal{E}}(p)\right|}$. Due to the homogeneity of $M,\left|\operatorname{ev}_{\mathcal{E}}(p)\right|$ is independent of $p$. Set

$$
\phi=\operatorname{ev}_{\mathcal{E}}(o),
$$

and $c_{j}=\left|\operatorname{ev}_{\mathcal{E}_{j}}(p)\right|, \phi_{j}=\operatorname{ev}_{\mathcal{E}_{j}}(o)$, where $j=1, \ldots, l$. Clearly, $\phi=\phi_{1}+\cdots+\phi_{l}$. Since $\phi_{j}$ are pairwise orthogonal, we get

$$
c^{2}=c_{1}^{2}+\cdots+c_{l}^{2},
$$

where $c$ is defined by (1.3) Let $\mathfrak{g}$ be the Lie algebra of $G$.

LEMMA 2.1. Set $\nu_{j}=\frac{c_{j}^{2}}{c^{2}}$, and let $s_{j}$ be the coefficient of the metric homothety for $\mathcal{E}_{j}, j=1, \ldots, l$. Then

$$
\begin{array}{r}
\nu_{1}+\cdots+\nu_{l}=1, \\
s^{2}=\nu_{1} s_{1}^{2}+\cdots+\nu_{l} s_{l}^{2} .
\end{array}
$$

Proof. The equality (2.3) follows from (2.2). For any $\xi \in \mathfrak{g}$, we have $d_{p} \iota(\xi(p))=$ $\frac{1}{c} \xi \operatorname{ev}_{\mathcal{E}}(p)$ and, according to (1.4),

$$
c s|\xi(o)|_{T_{o} M}=|\xi \phi|_{\mathcal{E}} \text {. }
$$

Similarly, $s_{j} c_{j}|\xi(o)|_{T_{o} M}=\left|\xi \phi_{j}\right|_{\mathcal{E}_{j}}$. Since $\xi \phi_{j} \in \mathcal{E}_{j}$,

$|\xi \phi|_{\mathcal{E}}^{2}=\left|\xi \phi_{1}+\cdots+\xi \phi_{l}\right|_{\mathcal{E}}^{2}=\left|\xi \phi_{1}\right|_{\mathcal{E}_{1}}^{2}+\cdots+\left|\xi \phi_{l}\right|_{\mathcal{E}_{l}}^{2}=\left(c_{1}^{2} s_{1}^{2}+\cdots+c_{l}^{2} s_{l}^{2}\right)|\xi(o)|_{T_{o} M}$.

Together with (2.5), this implies (2.4).

Let $\langle\rangle,, \widetilde{\langle,\rangle}$ be $G$-invariant inner products in $\mathcal{E}, \mathcal{S}$ and $\widetilde{\mathcal{S}}$ be the corresponding unit spheres in $\mathcal{E}$, respectively. We shall endow with the tilde the notation for relating objects. We assume additionally that there are $\tau_{j}>0, j=1, \ldots, l$, such that for all $u, v \in \mathcal{E}$

$$
\widetilde{\langle u, v\rangle}=\tau_{1}^{-1}\left\langle u_{1}, v_{1}\right\rangle+\cdots+\tau_{l}^{-1}\left\langle u_{l}, v_{l}\right\rangle,
$$

where $u_{j}, v_{j}$ are components of $u, v$ in the decomposition (1.6). The assumption holds if the summands in (1.6) are pairwise non-equivalent as $G$-modules. This is true if $M=S^{m}$.

LEMMA 2.2. For any $j=1, \ldots, l$, the following equalities hold:

$$
\begin{array}{r}
\tilde{c}_{j}^{2}=\tau_{j} c_{j}^{2}, \\
\tilde{\phi}_{j}=\tau_{j} \phi_{j}, \\
\tilde{s}_{j}=s_{j}=\sqrt{\frac{\lambda_{j}}{m},}
\end{array}
$$

and, moreover, $\tilde{s}^{2}=\frac{\tau_{1} c_{1}^{2}}{\tilde{c}^{2}} s_{1}^{2}+\cdots+\frac{\tau_{l} c_{l}^{2}}{\tilde{c}^{2}} s_{l}^{2}$, where $\tilde{c}^{2}=\tau_{1} c_{1}^{2}+\cdots+\tau_{l} c_{l}^{2}$.

Proof. Suppose $\langle u, v\rangle=\tau \widetilde{\langle u, v\rangle}$ for all $u, v \in \mathcal{E}$. Due to the equalities $u(o)=$ $\widetilde{\langle u, \tilde{\phi}\rangle}=\left\langle u, \frac{1}{\tau} \tilde{\phi}\right\rangle=\langle u, \phi\rangle$, we have $\tilde{\phi}=\tau \phi$ and $\tilde{c}^{2}=\widetilde{\tilde{\phi}}^{2}=\tau|\phi|^{2}=\tau c^{2}$ in this case. By (2.5),

$$
\begin{aligned}
& c s|\xi(o)|_{T_{o} M}=\frac{|\xi \phi|}{|\tilde{\tilde{\phi}}|} \\
& c \tilde{s}|\xi(o)|_{T_{o} M}=\mid
\end{aligned}
$$


for all $\xi \in \mathfrak{g}$. The equality $\tilde{\phi}=\tau \phi$ implies $\widetilde{\xi \tilde{\phi}}^{2}=\tau|\xi \phi|^{2}$ and, together with $\tilde{c}^{2}=\tau c^{2}$ and the equalities above, $\tilde{s}=s$. According to [10, Lemma 1], in the case of $L^{2}(M)$-norm and $\mathcal{E}$ irreducible we have $s=\sqrt{\frac{\lambda}{m}}$, where $\lambda$ is the eigenvalue of $-\Delta_{M}$ on $\mathcal{E}$ (in $\mathbf{1 0}$, it is assumed that $\mathcal{E} \perp \mathbf{1}$ but for the space of constant functions the equality $s=\sqrt{\frac{\lambda}{m}}$ is evidently true since $\lambda=s=0$ ). Since the invariant inner products on irreducible $G$-modules are pairwise proportional, the arguments above prove the first three equalities. The remaining ones follow from Lemma 2.1 and (2.2).

Corollary 2.3. Let $\lambda_{\min }$ and $\lambda_{\max }$ be the least and largest eigenvalues of $-\Delta_{M}$ in $\mathcal{E}$, respectively. Suppose $\lambda_{\min }<\lambda_{\max }$. Then

$$
\sqrt{\frac{\lambda_{\min }}{m}}<s<\sqrt{\frac{\lambda_{\max }}{m}} .
$$

Moreover, for any s satisfying this inequality there is an invariant Euclidean norm on $\mathcal{E}$ whose coefficient of metric homothety is equal to $s$.

Proof. If $\mathcal{E}_{j}$ contains a non-constant function, then $\lambda_{j}>0$ and, consequently, $\nu_{j}>0$. Thus the equality (2.3) implies the inequalities above. Since $\tau_{j}$ 's may be arbitrary positive numbers, the second assertion of the corollary follows from Lemma 2.2

The equivalent inequalities for $\mathrm{SO}(m+1)$-invariant Gaussian distributions $\mathcal{P}_{n}$ were stated without proof in Podkorytov's paper [16].

\section{The coefficients for the space of homogeneous polynomials}

We refer to [1, Chapter 5] for known facts on harmonic polynomials. In this section, we find the coefficients introduced in the previous one for the decomposition (1.1) and the following Euclidean norms: the first, | |, is the norm of $L^{2}\left(S^{m}\right)$ for the invariant probability measure and the second, $\widetilde{\mid} \mid$, is defined by

$$
\left.\widehat{\left\langle x^{\alpha}, x^{\beta}\right.}\right\rangle= \begin{cases}\alpha !, & \alpha=\beta \\ 0, & \alpha \neq \beta\end{cases}
$$

where $\alpha=\left(\alpha_{0}, \alpha_{1}, \ldots, \alpha_{m}\right), \alpha !=\alpha_{0} ! \ldots \alpha_{m}$ !, and $x^{\alpha}=x_{0}^{\alpha_{0}} \ldots x_{m}^{\alpha_{m}}$. A short straightforward computation shows that the formula

$$
\widetilde{\langle u, v\rangle}=u\left(\frac{\partial}{\partial x}\right) v
$$

defines the same inner product in $\mathcal{P}_{n}$ (notice that the right-hand part is constant). To the best of my knowledge, it was introduced in the the book $\mathbf{1 8}$ by E. Stein. The products are SO $(m+1)$-invariant. The Kostlan-Shub-Smale model corresponds to the Gaussian distribution whose density is proportional to $e^{-\widetilde{|u|}^{2}}$ on $\mathcal{P}_{n}$.

Let us use the notation of (1.1). On $S^{m}, \mathcal{H}_{j}$ is the eigenspace of the LaplaceBeltrami operator $\Delta_{S m}$ corresponding to the eigenvalue $-j(j+m-1)$. Set $\lambda_{j}=$ $j(j+m-1)$. It is known that $\operatorname{dim} \mathcal{P}_{n}=\left(\begin{array}{c}n+m \\ m\end{array}\right)$. According to (1.1),

$$
\operatorname{dim} \mathcal{H}_{n}=\left(\begin{array}{c}
n+m \\
m
\end{array}\right)-\left(\begin{array}{c}
n+m-2 \\
m
\end{array}\right)=\frac{(m+n-2) !(m+2 n-1)}{(m-1) ! n !}
$$


if $n \geq 2$. Clearly, $\operatorname{dim} \mathcal{H}_{0}=1$, $\operatorname{dim} \mathcal{H}_{1}=m+1$ and, moreover, the equalities hold for $n=0,1$ if we replace the factorials with $\Gamma$ and extend the right-hand side of (3.3) analytically. For short, set

$$
\varkappa(x)=|x|^{2}=x_{0}^{2}+x_{1}^{2}+\cdots+x_{m}^{2} .
$$

Lemma 3.1. Let $u \in \mathcal{H}_{j} \backslash\{0\}$ and $k \geq 0$ be integer. Then

$$
\frac{{\widetilde{\left|\varkappa^{k} u\right|}}^{2}}{|u|^{2}}=2^{k} k ! \prod_{i=1}^{j+k}(m+2 i-1)
$$

Proof. For $u \in \mathcal{P}_{j}$, let $u(x)=\sum_{|\alpha|=j} u_{\alpha} x^{\alpha}$, where $|\alpha|=\alpha_{0}+\cdots+\alpha_{m}$ and $x^{\alpha}=x_{0}^{\alpha_{0}} \cdots x_{m}^{\alpha_{m}}$, be its decomposition into the sum of monomials. By definition,

$$
\widetilde{|u|}^{2}=\sum_{|\alpha|=j} u_{\alpha}^{2} \alpha !
$$

If $u \in \mathcal{H}_{j}$, then its $L^{2}$-norm $|u|$ can be computed by a similar formula ([1, Theorem 5.14]):

$$
|u|^{2}=\frac{1}{(m+1)(m+3) \ldots(m+2 j-1)} \sum_{|\alpha|=j} u_{\alpha}^{2} \alpha ! .
$$

This proves the lemma in the case $k=0$. Thus we have a base for the induction on $k$. Let $\varphi$ be a smooth function on $\mathbb{R}$ and $v \in \mathcal{P}_{j}$. Using the equalities

$$
\begin{array}{r}
\Delta \varphi(\varkappa)=4 \varphi^{\prime \prime}(\varkappa) \varkappa+2(m+1) \varphi^{\prime}(\varkappa), \\
\langle\nabla \varphi(\varkappa), \nabla v\rangle=2 j \varphi^{\prime}(\varkappa) v,
\end{array}
$$

where $\Delta$ and $\nabla$ stand for the standard Euclidean operators on $\mathbb{R}^{m+1}$, we get

$$
\Delta(\varphi(\varkappa) v)=2\left(2 \varphi^{\prime \prime}(\varkappa) \varkappa+(m+2 j+1) \varphi^{\prime}(\varkappa)\right) v+\varphi(\varkappa) \Delta v .
$$

If $u \in \mathcal{H}_{j}$ and $\varphi(\varkappa)=\varkappa^{k}$, then

$$
\Delta\left(\varkappa^{k} u\right)=2 k(m+2 j+2 k-1) \varkappa^{k-1} u .
$$

By (3.2),

$$
{\widetilde{\mid \varkappa^{k} u}}^{2}=\left\langle\varkappa^{k} u, \varkappa^{k} u\right\rangle=\left\langle\varkappa^{k-1} \widetilde{u, \Delta\left(\varkappa^{k} u\right)}\right\rangle=2 k(m+2(j+k)-1){\widetilde{\varkappa^{k-1} u}}^{2} .
$$

This verifies the step of the induction and concludes the proof.

For the convenience of the reader, we collect the formulas for coefficients in the following proposition. The definitions of the parameters $c, s$ are given in (1.3), (1.4), respectively. The index $j$ corresponds to the summand $|x|^{n-j} \mathcal{H}_{j}$ in the decomposition (1.1). The coefficients $\nu_{j}$ and the rescaling factors $\tau_{j}$ are defined in Lemma 2.1 and by the formula (2.6), respectively. Further, | | is the norm of $L^{2}\left(S^{m}\right)$. The tilde distinguishes objects corresponding to the Kostlan-Shub-Smale model, in particular, $\widetilde{\|}$ is defined by (3.5). 
Proposition 3.1. We have

$$
\begin{array}{r}
c^{2}=\operatorname{dim} \mathcal{P}_{n}=\sum_{j \in J_{n}} c_{j}^{2}=\left(\begin{array}{c}
m+n \\
m
\end{array}\right), \\
c_{j}^{2}=\operatorname{dim} \mathcal{H}_{j}=\frac{(m+j-2) !(m+2 j-1)}{(m-1) ! j !}, \\
s_{j}^{2}=\frac{j(m+j-1)}{m}, \\
s^{2}=\frac{1}{c^{2}} \sum_{j \in J_{n}} c_{j}^{2} s_{j}^{2}=\frac{n(m+n+1)}{m+2},
\end{array}
$$

and $\nu_{j}=\frac{c_{j}^{2}}{c^{2}}$. Set $K_{n}=2^{-n} \Gamma\left(\frac{m+1}{2}\right)$. The coefficients $\tau_{j}$ for || and $\tilde{\|} \mid$ are subject to the formula

$$
\tau_{j}=\frac{K_{n}}{\Gamma\left(\frac{n-j+2}{2}\right) \Gamma\left(\frac{m+n+j+1}{2}\right)} .
$$

Furthermore, $\tilde{s}_{j}=s_{j}, \tilde{\nu}_{j}=n ! \tilde{c}_{j}^{2}$, where

$$
\begin{array}{r}
\tilde{c}_{j}^{2}=\tau_{j} c_{j}^{2}=\frac{K_{n}(m+j-2) !(m+2 j-1)}{(m-1) ! j ! \Gamma\left(\frac{n-j+2}{2}\right) \Gamma\left(\frac{m+n+j+1}{2}\right)}, \\
\tilde{c}^{2}=\frac{1}{n !}, \\
\tilde{s}^{2}=n .
\end{array}
$$

Proof. For any invariant finite dimensional subspace of $L^{2}(M)$ the squared norm of the evaluation functional at a point of $M$ is equal to the dimension of the space since the integral operator with the $\operatorname{kernel} \phi(p, q)=\left\langle\operatorname{ev}_{\mathcal{E}}(p), \operatorname{ev}_{\mathcal{E}}(q)\right\rangle$ is the orthogonal projection onto $\mathcal{E}$ and its trace is equal to $\int_{M} \phi(p, p) d p$. This proves the first equalities in (3.7) and (3.8) and, together with (??), (3.3), and (2.2) the remainder of (3.7) and (3.8).

Since $\mathcal{H}_{j}$ is the $\lambda_{j}$-eigenspace of $-\Delta_{S^{m}}$ with $\lambda_{j}=j(m+j-1)$, (3.9) is a consequence of Lemma 2.2,

According to (3.8) and (3.9), (3.10) is equivalent to the equality

$$
\sum_{j \in J_{n}} \frac{(m+j-2) !(m+2 j-1)}{(m-1) ! j !} \cdot \frac{j(m+j-1)}{m}=\left(\begin{array}{c}
m+n \\
m
\end{array}\right) \cdot \frac{n(m+n+1)}{m+2},
$$

where $m \geq 2$. Assuming $\left(\begin{array}{l}n \\ k\end{array}\right)=0$ for integer $k<0$ or $k>n$ (this is true for the analytic extension of $\left(\begin{array}{l}n \\ k\end{array}\right)$ on $k$ ), we may rewrite this equality as

$$
\sum_{j \in J_{n}}\left\{\left(\begin{array}{c}
m+j \\
m+1
\end{array}\right)+\left(\begin{array}{c}
m+j-1 \\
m+1
\end{array}\right)\right\}=\left(\begin{array}{c}
m+n+1 \\
m+2
\end{array}\right) .
$$

The formula above is equivalent to the following one, which is an easy consequence of the Pascal Triangle equality:

$$
\sum_{j=1}^{n}\left(\begin{array}{c}
m+j \\
m+1
\end{array}\right)=\left(\begin{array}{c}
m+n+1 \\
m+2
\end{array}\right)
$$


By definition, $\nu_{j}=\frac{c_{j}^{2}}{c^{2}}$ (see Lemma 2.1). The equality (3.11) follows from Lemma 3.1 with $k=\frac{n-j}{2}$. Since $\mathrm{SO}(m+1)$ is irreducible in $\mathcal{H}_{j}, \tilde{c}_{j}^{2}=\tau_{j} c_{j}^{2}$ and $\tilde{s}_{j}=s_{j}$ by Lemma 2.2

By $(3.1), \widetilde{\left\langle u, x_{0}^{n}\right\rangle}=n ! u(o)$ for all monomials $u \in \mathcal{P}_{n}$, where $o=(1,0, \ldots, 0)$. Hence $\tilde{\phi}(x)=\frac{1}{n !} x_{0}^{n}, \widetilde{\mid \tilde{\phi}}^{2}=\frac{1}{n !}$ and (3.12) follows.

Due to (2.5), we may compute $s$ applying to $\tilde{\phi}_{o}$ any vector field $\xi \in \operatorname{so}(m+1)$ such that $\xi(o) \neq 0$. Let $\xi=x_{1} \frac{\partial}{\partial x_{0}}-x_{0} \frac{\partial}{\partial x_{1}}$. Then $|\xi(o)|=1$ and we get

$$
\tilde{s}^{2}=\frac{{\widetilde{\xi \tilde{\phi}_{o}}}^{2}}{\tilde{c}^{2}}=\frac{1}{n !}\left|\widetilde{n x_{0}^{n-1} x_{1}}\right|^{2}=n .
$$

This concludes the proof of the proposition.

Equivalent forms of the equalities (3.4) and (3.13) are known from Kostlan's papers $1 \mathbf{1 2}$ and $\left[\mathbf{1 3}\right.$, respectively. In [16], Podkorytov stated the equality $s^{2}=$ $\frac{n(n+m+1)}{m+2}$ without proof.

\section{The scaling limit of the coefficients $\nu_{j}$ as $n \rightarrow \infty$}

Now, our aim is to find the scaling limit of the coefficients $\tilde{\nu}_{j}=\frac{\tilde{c}_{j}^{2}}{\tilde{c}^{2}}$. The coefficients $c_{j}^{2}$ and $\tau_{j}$ (see (3.7) and (3.11), respectively) admit the evident extensions on $j$ onto $\mathbb{C}$, which are entire functions which we denote as $c^{2}(\zeta)$ and $\tau(\zeta)$. The function $c^{2}(\zeta)$ is a polynomial of degree $m-1$. Thus,

$$
\begin{array}{r}
\tilde{c}^{2}(\zeta)=\tau(\zeta) c^{2}(\zeta), \\
\tilde{\nu}(\zeta)=n ! \tilde{c}^{2}(\zeta)
\end{array}
$$

are entire functions. In this section, $c^{2}$ and $\tilde{c}^{2}$ denote the extensions (thus they are not the sums of $c_{j}^{2}$ and $\tilde{c}_{j}^{2}$ as in the previous one). These functions depend on $m$ and $n$ which we omit in the notation. Note that $\tau, c^{2}$, and $\tilde{\nu}$ are positive on the interval $(0, n)$.

LEMMA 4.1. The function $\ln \tilde{\nu}$ is strictly concave on the interval $(0, n)$ and has the unique maximum on it.

Proof. We have $\ln c^{2}(x)^{\prime \prime}<0$ because $c^{2}$ is a product of linear functions, for $\ln \tau$ the same is true since $(\ln \Gamma(x))^{\prime \prime}>0$. Hence $\ln \tilde{\nu}$ is strictly concave.

Suppose that

$$
\tilde{\nu}(x+2)=\tilde{\nu}(x) \text { for some } x \in(0, n-2) .
$$

Then $\tilde{\nu}$ has a critical point

$$
x_{c} \in(x, x+2)
$$

which necessarily is unique and corresponds to the global maximum on $(0, n)$. Thus, it is sufficient to prove 4.1). Set

$$
\rho_{n}(x)=\frac{\tilde{\nu}(x+2)}{\tilde{\nu}(x)},
$$

where $x$ runs over $(0, n-2)$. The condition (4.1) is equivalent to

$$
\rho_{n}(x)=1 \text { for some } x \in(0, n-2) \text {. }
$$


According to (3.8) and (3.11), $\frac{\tau(x+2)}{\tau(x)}=\frac{n-x}{n+x+m+1}$ and

$$
\rho_{n}(x)=\left(1+\frac{m-2}{x+1}\right)\left(1+\frac{m-2}{x+2}\right)\left(1+\frac{2}{x+\frac{m-1}{2}}\right) \frac{n-x}{n+x+m+1} .
$$

Due to (1.8), $\rho_{n}(0)=\frac{2 m n}{m+n+1}>1$. Assuming $n>3$ and applying (1.8) again, we get

$$
\rho_{n}(n-2)=\frac{2(n+m-3)(n+m-2)}{n(n-1)(2 n+m-5)}<\frac{4(n+m-3)}{n(2 n+m-5)} \leq \frac{n+m-3}{2 n+m-5}<1 .
$$

If $n=3$, then $m=2$ and $\rho_{n}(n-2)=\frac{2}{3}$. Since $n \geq 3$ by (1.8), this proves (4.2) and consequently the lemma.

Set

$$
\begin{array}{r}
\mu_{n}=\sqrt{(m-1) n}, \\
\bar{\nu}_{n}=\tilde{\nu}\left(x_{c}\right),
\end{array}
$$

where $x_{c}$ is the critical point of $\tilde{\nu}$ (hence $\bar{\nu}_{n}$ is the maximum of $\tilde{\nu}(x)$ on $[0, n]$ ).

In the following theorem, we assume that $\tilde{\nu}$ is a function on $(0, \infty)$ which vanishes outside $(0, n)$.

THEOREM 4.2. If $n$ is sufficiently large, then

$$
\mu_{n}-\frac{m+1}{2}<x_{c}<\mu_{n}+2 .
$$

For any $t>0$

$$
\lim _{n \rightarrow \infty} \frac{\tilde{\nu}\left(\mu_{n} t\right)}{\bar{\nu}_{n}}=\left(t^{2} e^{1-t^{2}}\right)^{\frac{m-1}{2}},
$$

where the sequence on the left converges uniformly on $(0, \infty)$. Moreover,

$$
\bar{\nu}_{n}=\frac{A_{m}}{\sqrt{n}}(1+o(1))
$$

as $n \rightarrow \infty$, where $A_{m}=\frac{2 \sqrt{2}}{\Gamma\left(\frac{m}{2}\right)}\left(\frac{m-1}{2 e}\right)^{\frac{m-1}{2}}$.

Proof. Setting $x=a \sqrt{n}$ in factors of $\rho_{n}$, by the straightforward computation we get

$$
\rho_{n}(a \sqrt{n})=1+\left(\frac{2(m-1)}{a}-2 a\right) \frac{1}{\sqrt{n}}+O\left(\frac{1}{n}\right) .
$$

Therefore,

$$
\rho_{n}(a \sqrt{n})=1+O\left(\frac{1}{n}\right) \Longleftrightarrow a=\sqrt{m-1}
$$

Thus $\mu_{n}$ can be considered as an approximation of $x_{c}$. According to the calculation above,

$$
\rho_{n}\left(\mu_{n}\right)=1-\frac{2(m+1)}{n}+O\left(n^{-\frac{3}{2}}\right)
$$

It follows that

$$
\rho_{n}\left(\mu_{n}\right)<1
$$

for sufficiently large $n$. The function $\rho_{n}$ is positive, decreasing, and convex since each factor in (4.3) possesses these properties (the factors are convex because all 
of them may be written as $\pm 1+\frac{a}{x+b}$, where $a>0$ ). Thus (4.10) gives an upper bound for $x_{c}$ :

$$
x_{c}<\mu_{n}+2 .
$$

To find a lower bound, we do one step of the Newton method:

$$
\eta_{n}=\mu_{n}-\frac{\rho_{n}\left(\mu_{n}\right)-1}{\rho_{n}^{\prime}\left(\mu_{n}\right)}
$$

Since $\rho_{n}$ is convex and decreasing, $\rho_{n}\left(\eta_{n}\right)>1$. Hence $x_{c}>\eta_{n}$. We shall replace $\eta_{n}$ with a simpler term which is asymptotic to it as $n \rightarrow \infty$. Due to (4.9), it is sufficient to do this for $\rho_{n}^{\prime}\left(\mu_{n}\right)$. Let us consider $\left(\ln \rho_{n}\right)^{\prime}$. For the first three factors in (4.3) we may use the equality $\ln \left(1+\frac{a}{x+b}\right)^{\prime}=-\frac{a}{(x+a)(x+a+b)}=-\frac{a}{x^{2}}+O\left(x^{-3}\right)$ as $x \rightarrow \infty$. The logarithmic derivative of the forth one is equal to $\frac{2 n+m+1}{(n-x)(n+x+m+1)}$. Setting $x=\mu_{n}$, we get

$$
\frac{\rho_{n}^{\prime}\left(\mu_{n}\right)}{\rho_{n}\left(\mu_{n}\right)}=-\left(2 \cdot \frac{m-2}{\mu_{n}^{2}}+\frac{2}{\mu_{n}^{2}}+\frac{2}{n}\right)+O\left(n^{-\frac{3}{2}}\right)=-\frac{4}{n}+O\left(n^{-\frac{3}{2}}\right) .
$$

Together with (4.4) and (4.9), this implies

$$
\rho_{n}^{\prime}\left(\mu_{n}\right)=-\frac{4}{n}+O\left(n^{-\frac{3}{2}}\right) .
$$

Therefore,

$$
\mu_{n}-\eta_{n}=\frac{\rho_{n}\left(\mu_{n}\right)-1}{\rho_{n}^{\prime}\left(\mu_{n}\right)}=\frac{m+1}{2}+O\left(n^{-\frac{3}{2}}\right) .
$$

Furthermore,

$$
\lim _{n \rightarrow \infty} n^{\frac{3}{2}}\left(\rho_{n}\left(\mu_{n}-\frac{m+1}{2}\right)-1\right)=\frac{m(m+1)}{\sqrt{m-1}} .
$$

Hence $\rho_{n}\left(\mu_{n}-\frac{m+1}{2}\right)>1$ for sufficiently large $n$. This proves 4.6).

The following equalities can be proved by a computation:

$$
\begin{array}{r}
\lim _{n \rightarrow \infty} \rho_{n}\left(\mu_{n} x\right)=1, \\
\lim _{n \rightarrow \infty} \mu_{n} \ln \rho_{n}\left(\mu_{n} x\right)=2(m-1)\left(\frac{1}{x}-x\right),
\end{array}
$$

and the convergence is locally uniform on $(0, \infty)$. The sequence of piecewise constant functions defined by the equality

$$
f_{n}(\xi)=\mu_{n} \ln \rho_{n}(i)
$$

for $\xi \in\left(\frac{i-2}{\mu_{n}}, \frac{i}{\mu_{n}}\right]$, where $i \in J_{n}$, converges to $2(m-1)\left(\frac{1}{\xi}-\xi\right)$ locally uniformly on $(1, t)$. Thus,

$$
\begin{array}{r}
\int_{1}^{t} f_{n}(\xi) d \xi=\frac{2}{\mu_{n}} \sum_{\substack{i \in J_{n}, i<\mu_{n} t}} \mu_{n} \ln \rho_{n}(i)+O\left(n^{-\frac{1}{2}}\right)=2 \ln \prod_{\substack{i \in J_{n}, i<\mu_{n} t}} \rho_{n}(i)+O\left(n^{-\frac{1}{2}}\right) \\
\lim _{n \rightarrow \infty} \ln \frac{\tilde{\nu}\left(\mu_{n} t\right)}{\tilde{\nu}\left(\mu_{n}\right)}=\lim _{n \rightarrow \infty} \ln \prod_{\substack{\mu_{n}<i<\mu_{n} t, n-i \text { even }}} \rho_{n}(i)=\frac{1}{2} \lim _{n \rightarrow \infty} \int_{1}^{t} f_{n}(\xi) d \xi \\
=(m-1) \int_{1}^{t}\left(\frac{1}{\xi}-\xi\right) d \xi=\frac{m-1}{2}\left(1+2 \ln t-t^{2}\right) .
\end{array}
$$


V. GICHEV

Here and in what follows, we use Lebesgue's Dominated Convergence Theorem with the majorant of the type $K e^{-\eta t}$. The convergence is locally uniform. Due to (4.6),

$$
\lim _{n \rightarrow \infty} \frac{\tilde{\nu}\left(\mu_{n}\right)}{\bar{\nu}_{n}}=\lim _{n \rightarrow \infty} \frac{\tilde{\nu}\left(\mu_{n}\right)}{\tilde{\nu}\left(x_{c}\right)}=1 .
$$

Therefore, $\lim _{n \rightarrow \infty} \frac{\tilde{\nu}\left(\mu_{n} t\right)}{\bar{\nu}_{n}}=\left(t^{2} e^{1-t^{2}}\right)^{\frac{m-1}{2}}$ for any $t>1$. The arguments above with minor changes can be extended onto the case $0<t \leq 1$. Thus (4.7) holds for all $t>0$. The sequence $\frac{\tilde{\nu}\left(\mu_{n} t\right)}{\bar{\nu}_{n}}$ converges uniformly on any compact interval in $(0, \infty)$. Since $\ln \tilde{\nu}\left(\mu_{n} t\right)$ is concave and $\tilde{\nu}\left(\mu_{n} t\right)$ is positive and has maximum near 1 , this implies the uniform convergence on $(0, \infty)$.

Furthermore,

$\lim _{n \rightarrow \infty} \frac{2}{\mu_{n}} \sum_{j \in J_{n}}\left(\frac{j^{2}}{\mu_{n}^{2}} e^{1-\frac{j^{2}}{\mu_{n}^{2}}}\right)^{\frac{m-1}{2}}=\int_{0}^{\infty}\left(t^{2} e^{1-t^{2}}\right)^{\frac{m-1}{2}} d t=\frac{\Gamma\left(\frac{m}{2}\right)}{2 \sqrt{e}}\left(\frac{2 e}{m-1}\right)^{\frac{m}{2}}$.

On the other hand, the equality $\sum_{j \in J_{n}} \tilde{\nu}_{j}=1$ which is true by (2.3), (4.7), and Lebesgue's Dominated Convergence Theorem imply

$$
\lim _{n \rightarrow \infty} \bar{\nu}_{n} \sum_{i \in J_{n}}\left(\frac{j^{2}}{\mu_{n}^{2}} e^{1-\frac{j^{2}}{\mu_{n}^{2}}}\right)^{\frac{m-1}{2}}=1 .
$$

Computing the ratio of the left-hand parts of the equalities above, we get

$$
\lim _{n \rightarrow \infty} \bar{\nu}_{n} \mu_{n}=\frac{4 \sqrt{e}}{\Gamma\left(\frac{m}{2}\right)}\left(\frac{m-1}{2 e}\right)^{\frac{m}{2}}=A_{m} \sqrt{m-1} .
$$

This proves (4.8) and the theorem.

Actually, $\mu_{n}-\frac{m-1}{2}$ is a better approximation for $x_{c}$ than $\mu_{n}$ or $\eta_{n}$ since it is the center of the interval $\left(\eta_{n}, \eta_{n}+2\right)$ and $\eta_{n}$ is close to the solution of the equation $\rho_{n}(x)=1$.

The constant $A_{m}$ in (4.8) decreases when $m$ grows and $\lim _{m \rightarrow \infty} A_{m}=\frac{2}{\sqrt{\pi}}$. Since $m \geq 2$, this implies the inequalities

$$
\frac{2}{\sqrt{\pi}}<A_{m} \leq \frac{2}{\sqrt{e}}
$$

We omit the proof which is standard.

Modifying the arguments above slightly, it is possible to find an upper bound for the ratio $\frac{\tilde{\nu}(j)}{\tilde{\nu}\left(\mu_{n}\right)}$. Then

Proposition 4.1. Set $j_{n}=\min \left\{j \in J_{n}: j>\mu_{n}\right\}$ and let $j \in J_{n}, j>j_{n}$.

$$
\frac{\tilde{\nu}(j+2)}{\tilde{\nu}\left(j_{n}+2\right)}<\frac{j^{m-1}}{j_{n}^{m-1}} e^{\frac{j_{n}^{2}-j^{2}}{2 n}} .
$$

Proof. Since $\rho_{n}(x)$ decreases on $(0, n)$,

$$
\int_{j_{n}}^{j} \ln \rho_{n}(x) d x>2 \sum_{\substack{i \in J_{n}, i<j}} \ln \rho_{n}(i+2)=2 \ln \frac{\tilde{\nu}(j+2)}{\tilde{\nu}\left(j_{n}+2\right)} .
$$


The inequality $\ln (1+x)<x$ and (4.3) imply

$$
\ln \rho_{n}(x)<\frac{m-2}{x+1}+\frac{m-2}{x+2}+\frac{4}{2 x+m-1}-\ln \frac{n+x+m+1}{n-x} .
$$

Due to the evident inequality $\frac{m-2}{x+1}+\frac{m-2}{x+2}+\frac{2}{x+\frac{m-1}{2}}<\frac{2(m-1)}{x}$, for the integral of the sum of the first three terms we have the upper bound

$$
2(m-1) \int_{j_{n}}^{j} \frac{d x}{x}=2(m-1) \ln \frac{j}{j_{n}} .
$$

If $0<t<1$, then $\ln \frac{1+t}{1-t}>2 t$. Setting $t=\frac{x}{n}$, we get the inequality $\ln \frac{n+x+m+1}{n-x}>$ $\frac{2 x}{n}$. Therefore,

$$
\int_{j_{n}}^{j} \ln \frac{n+x+m+1}{n-x} d x>\frac{j^{2}-j_{n}^{2}}{n} .
$$

Thus, $\int_{j_{n}}^{j} \ln \rho_{n}(x) d x<2(m-1) \ln \frac{j}{j_{n}}+\frac{j_{n}^{2}-j^{2}}{n}$. Together with 4.18), this implies (4.17).

\section{Approximation by polynomials of lower degree}

Let $x$ be the Kostlan-Shub-Smale random polynomial in $\mathcal{P}_{n}$. In this section, we show that $x$ admits a good approximation in Sobolev spaces on $S^{m}$ by polynomials of degree $l_{n} \sim C \sqrt{n \ln n}$, where $C$ depends on $m$ and on the order of the Sobolev space. We estimate the expectation of $\frac{\operatorname{dist}_{X}\left(x, \mathcal{P}_{l_{n}}\right)}{\|x\|}$ and the probability of the inequality $\operatorname{dist}_{X}\left(x, \mathcal{P}_{l_{n}}\right)<\varepsilon\|x\|_{X}$, where $X$ is the Sobolev space $H^{q}=H^{q}\left(S^{m}\right)$, $q \geq 0$, with the norm

$$
|x|_{q}=\left(\left|x_{0}\right|^{2}+\sum_{j=1}^{\infty} j^{2 q}\left|x_{j}\right|^{2}\right)^{\frac{1}{2}} .
$$

If $j>m-1$, then $j^{2}<\lambda_{j}<2 j^{2}$, where $\lambda_{j}=j(j+m-1)$ is the $j$ th eigenvalue of the Laplace-Beltrami operator $\Delta_{S m}$ on $S^{m}$. Hence $|x|_{q}$ is equivalent to the norm

$$
\left(\left|x_{0}\right|^{2}+\left|\left(-\Delta_{S^{m}}\right)^{\frac{q}{2}} u\right|^{2}\right)^{\frac{1}{2}}=\left(\left|x_{0}\right|^{2}+\sum_{j=0}^{\infty} \lambda_{j}^{q}\left|x_{j}\right|^{2}\right)^{\frac{1}{2}}
$$

Clearly, $H^{0}=L^{2}\left(S^{m}\right)$.

LEMMA 5.1. For sufficiently large $n$ the function $\alpha(x)=x^{2 q} \tau(x)$ strictly decreases on the interval $(\sqrt{2 q n}+2, n)$.

Proof. Since $\ln \Gamma$ is strictly convex, the function $\ln \tau(x)$ is strictly concave on $(0, n)$. Hence the same is true for $\ln \alpha(x)$. Set

$$
\varphi(x)=\frac{\alpha(x+2)}{\alpha(x)}=\left(1+\frac{2}{x}\right)^{2 q} \frac{n-x}{n+x+m+1} .
$$

Let $q>0$. Then $\varphi(x) \rightarrow \infty$ as $x \rightarrow 0$. Hence the inequality $\varphi(a)<1$ for $a \in(0, n)$ implies $\varphi(b)=1$ for some $b \in(0, a)$. Then $\alpha$ has a critical point in $(b, b+2)$. Thus, $\alpha$ decreases on $(a+2, n)$ if $\varphi(a)<1$. A calculation shows that

$$
\varphi(\sqrt{2 q n})=1-\left(m+1+\frac{1}{q}\right) \frac{1}{n}+O\left(n^{-\frac{3}{2}}\right) .
$$


Hence $\varphi(\sqrt{2 q n})<1$ for large $n$.

If $q=0$, then $\alpha(x)=\tau(x)$. Since $(\ln \tau)^{\prime}(x)=\frac{1}{2} \Psi\left(\frac{n-x+2}{2}\right)-\frac{1}{2} \Psi\left(\frac{n+x+m+1}{2}\right)$, where $\Psi=(\ln \Gamma)^{\prime}$, and $(\ln \Gamma)^{\prime \prime}(x)>0$, we get $\tau^{\prime}(x)<0$ for all $x>0$. This concludes the proof of the lemma.

LEMMA 5.2. Let $x=x_{1} \oplus x_{2} \oplus x_{3}$ correspond to the decomposition $\mathbb{R}^{d}=$ $\mathbb{R}^{d_{1}} \oplus \mathbb{R}^{d_{2}} \oplus \mathbb{R}^{d_{3}}, a>-d_{1}$, and $d_{2}>2$. Then

$$
\begin{array}{r}
\int_{S^{d-1}}\left|x_{1}\right|^{a} d x=\frac{\Gamma\left(\frac{d_{1}+a}{2}\right) \Gamma\left(\frac{d}{2}\right)}{\Gamma\left(\frac{d+a}{2}\right) \Gamma\left(\frac{d_{1}}{2}\right)}, \\
\int_{S^{d-1}} \frac{\left|x_{1}\right|^{2}}{\left|x_{2}\right|^{2}} d x=\frac{d_{1}}{d_{2}-2} .
\end{array}
$$

We omit the standard proof.

THEOREM 5.3. Let $l_{n}$ be a sequence of positive integers such that $l_{n}<n$,

$$
\begin{array}{r}
\limsup _{n \rightarrow \infty} \frac{l_{n}}{n}<1, \\
\lim _{n \rightarrow \infty} n^{m+2 q} e^{-\frac{l_{n}^{2}}{n}}=0 .
\end{array}
$$

Suppose that $t_{n}>0$ satisfy the conditions

$$
\lim _{n \rightarrow \infty} n^{m} t_{n}^{-4}=\lim _{n \rightarrow \infty} t_{n}^{4} n^{2 q} e^{-\frac{l_{n}^{2}}{n}}=0
$$

Then there exist $A, B>0$, where $A$ depends only on $q$ and $B$ depends only on the sequence $l_{n}$, such that for

$$
\begin{array}{r}
\eta_{n}=A n^{\frac{m}{2}} t_{n}^{-2}, \\
\varepsilon_{n}=B t_{n} n^{\frac{q}{2}} e^{-\frac{l_{n}^{2}}{4 n}}
\end{array}
$$

we have $\lim _{n \rightarrow \infty} \varepsilon_{n}=\lim _{n \rightarrow \infty} \eta_{n}=0$ and for any sufficiently large $n$ the inequality

$$
\operatorname{dist}_{H^{q}}\left(x, \mathcal{P}_{l_{n}}\right)<\varepsilon_{n}|x|_{q},
$$

holds for the random Kostlan-Shub-Smale polynomial $x \in \mathcal{P}_{n}$ with the probability greater than $1-\eta_{n}$.

Proof. First of all, we notice that (5.5) implies $\varepsilon_{n}, \eta_{n} \rightarrow 0$ as $n \rightarrow \infty$.

Let $J_{n}$ be defined by (1.2). We consider the partition of $J_{n}$ by the following subsets of $[0, n]: I_{1}=[\sqrt{2 q n}, a \sqrt{n}]$, where $a>\sqrt{2 q}, I_{3}=\left[l_{n}, n\right]$, where $l_{n}>a \sqrt{n}$, and $I_{2}=[0, n] \backslash\left(I_{1} \cup I_{3}\right)$. Set

$$
\mathcal{P}_{n}=\mathcal{U}_{n} \oplus \mathcal{Z}_{n} \oplus \mathcal{V}_{n}
$$

where each summand is the sum of the spaces $\mathcal{H}_{j}$ with $j$ running over the intersection of $J_{n}$ with one of the sets $I_{1}, I_{2}, I_{3}$, respectively. For $x \in \mathcal{P}_{n}$, let $x=u+z+v$ be the corresponding decomposition of $x$. Then

$$
|v|_{q}=\operatorname{dist}_{H^{q}}\left(x, \mathcal{P}_{l_{n}}\right)
$$

since the decomposition (1.1) is orthogonal in the involved inner products. We shall estimate the probability of the inequality $|v|<\varepsilon_{n}|x|$ reducing it to the inequality 
$\widetilde{|v|}<t_{n} \widetilde{|u|}$ and estimating the expectation of the ratio $\widetilde{|v|}^{2} / \widetilde{|u|}^{2}$. Since it is homogeneous of degree 0, its distribution for the Kostlan-Shub-Smale model and for the uniform distribution in the unit sphere $\tilde{\mathcal{S}}$ coincide. By (5.2),

$$
\mathrm{E}\left(\widetilde{|v|}^{2} / \widetilde{|u|}^{2}\right)=\frac{\operatorname{dim} \mathcal{V}_{n}}{\operatorname{dim} \mathcal{U}_{n}-2}
$$

According to the decomposition (1.1),

$$
\operatorname{dim} \mathcal{U}_{n} \geq \operatorname{dim} \mathcal{P}_{[a \sqrt{n}]-1}-\operatorname{dim} \mathcal{P}_{[\sqrt{2 q n}]} .
$$

Replacing $\mathcal{V}_{n}$ with $\mathcal{P}_{n}$ in (5.8), we get

$$
\limsup _{n \rightarrow \infty} n^{-\frac{m}{2}} \mathrm{E}\left(\widetilde{|v|}^{2} / \widetilde{u u}^{2}\right) \leq \lim _{n \rightarrow \infty} \frac{\operatorname{dim} \mathcal{P}_{n}}{n^{\frac{m}{2}} \operatorname{dim} \mathcal{U}_{n}}=\frac{1}{a^{m}-(2 q)^{\frac{m}{2}}} .
$$

Set

$$
\tilde{V}_{t, l}=\{x \in \tilde{\mathcal{S}}: \widetilde{|v|}>t \widetilde{|u|}\} .
$$

Let $\tilde{\sigma}$ be the invariant probability measure on $\tilde{\mathcal{S}}$. Due to the Chebyshev inequality,

$$
A>\frac{1}{a^{m}-(2 q)^{\frac{m}{2}}} \Longrightarrow \tilde{\sigma}\left(\tilde{V}_{t_{n}, l_{n}}\right)<A n^{\frac{m}{2}} t_{n}^{-2}
$$

for all sufficiently large $n$. By (5.5), $\tilde{\sigma}\left(\tilde{V}_{t_{n}, l_{n}}\right) \rightarrow 0$ as $n \rightarrow \infty$. Let $a>2^{\frac{1}{m}} \sqrt{2 q}$. Then $a^{m}-(2 q)^{\frac{m}{2}}>(2 q)^{\frac{m}{2}}$. Thus for $A=(2 q)^{-\frac{m}{2}}$ the inequality on the right of (5.9) holds for all sufficiently large $n$.

Let $\alpha$ be as Lemma 5.1. Then $\alpha$ decreases on $(\sqrt{2 q n}+2, n)$ and

$$
\begin{aligned}
|v|_{q}^{2} & =\sum_{j \in J_{n} \cap I_{3}} \alpha(j){\widetilde{\mid v_{j}}}^{2}, \\
|u|_{q}^{2} & =\sum_{j \in J_{n} \cap I_{1}} \alpha(j){\widetilde{\mid u_{j}}}^{2},
\end{aligned}
$$

where the indices correspond to the decomposition (1.1), Therefore,

$$
\begin{gathered}
|v|_{q}^{2}<\left.\alpha\left(l_{n}\right) \widetilde{|v|}\right|^{2}, \\
|u|_{q}^{2}>\alpha(a \sqrt{n}) \mid{ }_{|u|}^{2}
\end{gathered}
$$

if $v \neq 0$ and $u \neq 0$ (we shall assume this in the sequel since this evidently does not affect the result). Hence $x \notin \tilde{V}_{t_{n}, l_{n}}$ implies

$$
|v|_{q}^{2}<t_{n}^{2} \frac{\alpha\left(l_{n}\right)}{\alpha(a \sqrt{n})}|u|_{q}^{2}=t_{n}^{2}\left(\frac{l_{n}^{2}}{a^{2} n}\right)^{q} \frac{\tau\left(l_{n}\right)}{\tau(a \sqrt{n})}|u|_{q}^{2} .
$$

The ratio $\frac{\tau\left(l_{n}\right)}{\tau(a \sqrt{n})}$ can be estimated as in Proposition 4.1. The arguments which prove the inequality (4.19) show that

$$
\ln \frac{\tau\left(l_{n}+2\right)}{\tau(a \sqrt{n}+2)}<-\int_{a \sqrt{n}}^{l_{n}} \ln \frac{n+x+m+1}{n-x} d x<\frac{a^{2}}{2}-\frac{l_{n}^{2}}{2 n} .
$$

It follows from the equality $\frac{\tau(x+2)}{\tau(x)}=\frac{n-x}{n+x+m+1}$ that $\lim _{n \rightarrow \infty} \frac{\tau(a \sqrt{n}+2)}{\tau(a \sqrt{n})}=1$. Due (5.3), for some $b>1$

$$
\limsup _{n \rightarrow \infty} \frac{\tau\left(l_{n}+2\right)}{\tau\left(l_{n}\right)}>\frac{1}{b}
$$


Hence

$$
\limsup _{n \rightarrow \infty} \frac{\tau\left(l_{n}\right) e^{\frac{l_{n}^{2}}{2 n}}}{\tau(a \sqrt{n})}=\limsup _{n \rightarrow \infty} \frac{\tau\left(l_{n}+2\right) e^{\frac{l_{n}^{2}}{2 n}}}{\tau(a \sqrt{n}+2)} \frac{\tau\left(l_{n}\right)}{\tau\left(l_{n}+2\right)} \frac{\tau(a \sqrt{n}+2)}{\tau(a \sqrt{n})}<b e^{\frac{a^{2}}{2}}
$$

If $q=0$, then $\left(\frac{l_{n}^{2}}{a^{2} n}\right)^{q}=1$ and we may choose arbitrary $a>0$. Thus, the inequality

$$
|v|_{q}^{2}<B t_{n}^{2} n^{q} e^{-\frac{l_{n}^{2}}{2 n}}|u|_{q}^{2}
$$

holds for all sufficiently large $n$ if $B=2 b$ and $x \notin \tilde{V}_{t_{n}, l_{n}}$ by (5.10) and (5.12) (we put $a=\sqrt{2 \ln 2}$ ). Let $q>0$. The evident inequality $\frac{l_{n}^{2}}{n}<n$ implies $\left(\frac{l_{n}^{2}}{a^{2} n}\right)^{q}<a^{-2 q} n^{q}$. By (5.10) and the inequalities above, (5.13) is true provided that

$$
B>a^{-2 q} b e^{\frac{a^{2}}{2}} \text {. }
$$

Set $a=2 \sqrt{q}$. Then $a^{-2 q} e^{\frac{a^{2}}{2}}=e^{2 q\left(1-\ln 2-\frac{1}{2} \ln q\right)}$. The function on the right attains its maximal value at $q=\frac{e}{4}$. It is approximately equal to $1.97<2$. Thus the setting $B=2 b$ satisfies (5.13) for $x \notin \tilde{V}_{t_{n}, l_{n}}$ and all $q \geq 0$ if $n$ is sufficiently large. This concludes the proof of the theorem.

REMARK 5.4. The sequence $t_{n}=n^{\frac{m}{8}-\frac{q}{4}} e^{\frac{l_{n}^{2}}{8 n}}$ satisfies (5.5) if (5.4) is true. For this choice of $t_{n}, \eta_{n}$ is proportional to $\varepsilon_{n}^{2}$. If $l_{n}>\alpha n$ for some $\alpha \in(0,1)$, then both $\varepsilon_{n}$ and $\eta_{n}$ decrease exponentionally. On the other hand, the expression under the limit in (5.4) is equal to $\varepsilon_{n}^{4} \eta^{2}$ up to a multiplicative constant depending only on $m$ and $q$. Hence neither $\varepsilon_{n}^{4}$ nor $\eta_{n}^{2}$ can decay faster than $n^{m+2 q} e^{-\frac{l_{n}^{2}}{n}}$.

If $l_{n}=\sqrt{(m+2 q+1) n \ln n}$, then $n^{m+2 q} e^{-\frac{l_{n}^{2}}{n}}=\frac{1}{n}$. For $t_{n}=n^{a}$ we have $\eta_{n}=A n^{\frac{m}{2}-2 a}, \varepsilon_{n}=B n^{a-\frac{m+1}{4}}$, and (5.5) is true if $m<4 a<m+1$.

Let $I$ be an interval in $(0, \infty)$ which may be infinite. Set $I_{n}=\mu_{n} I$ and let $\pi_{n}$ be the orthogonal projection onto the sum of the spaces $\mathcal{H}_{j}$ such that $j \in I_{n} \cap J_{n}$.

Proposition 5.1. Let $u$ be a random polynomial uniformly distributed in the unit sphere $\widetilde{\mathcal{S}} \subseteq \mathcal{P}_{n}$ for the norm $\widetilde{\|}$. Then

$$
\lim _{n \rightarrow \infty} \frac{\mathrm{E}\left(\left|\pi_{n} u\right|^{2}\right)}{\mathrm{E}\left(|u|^{2}\right)}=\frac{1}{A} \int_{I}\left(t^{2} e^{1-t^{2}}\right)^{\frac{m-1}{2}} d t
$$

where $A=\int_{0}^{\infty}\left(t^{2} e^{1-t^{2}}\right)^{\frac{m-1}{2}} d t=\frac{\Gamma\left(\frac{m}{2}\right)}{2 \sqrt{e}}\left(\frac{2 e}{m-1}\right)^{\frac{m}{2}}$ and, moreover, $\mathrm{E}\left(|u|^{2}\right)=\frac{m !}{(n+m) !}$.

Proof. Applying (5.1), (3.7), and (3.8), we get

$$
\begin{aligned}
\mathrm{E}\left(\left|\pi_{n} u\right|^{2}\right)=\sum_{j \in I_{n} \cap J_{n}} \int_{\widetilde{S}}\left|u_{j}\right|^{2} d u=\sum_{j \in I_{n} \cap J_{n}} \tau_{j} \int_{\widetilde{S}}{\widetilde{u_{j} \mid}}^{2} d u & =\sum_{j \in I_{n} \cap J_{n}} \frac{\tau_{j} c_{j}^{2}}{c^{2}} \\
& =\frac{\tilde{c}^{2}}{c^{2}} \sum_{j \in I_{n} \cap J_{n}} \tilde{\nu}_{j} .
\end{aligned}
$$

Setting $I=(0, \infty)$, we get $\mathrm{E}\left(|u|^{2}\right)=\frac{\tilde{c}^{2}}{c^{2}}=\frac{m !}{(n+m) !}$ according to (2.3), (3.7), and (3.12). As in Theorem 4.2, we may treat the sum as the integral of piecewise constant functions. Proposition 4.1 implies the existence of a common majorant for them. This proves the convergence of integrals as $n \rightarrow \infty$ and the proposition. 
In particular, for $I=(t, \infty)$ we have the integral $\int_{t}^{\infty}\left(\xi^{2} e^{1-\xi^{2}}\right)^{\frac{m-1}{2}} d \xi$. Using the elementary inequality $t^{2} e^{1-t^{2}}<e^{-(t-1)^{2}}$, which holds if $t>0$ and $t \neq 1$, and the standard estimate of the Gaussian integral, we get the following upper bound for the expectation of the distance in $L^{2}\left(S^{m}\right)$ from the random uniformly distributed polynomial in $\tilde{\mathcal{S}} \subset \mathcal{P}_{n}$ to the space $\mathcal{P}_{l_{n}}$ which holds if $t>1, l_{n}>t \mu_{n}$, $l_{n} \in J_{n}$, and $n$ is sufficiently large:

$$
\mathrm{E}\left(\operatorname{dist}\left(u, \mathcal{P}_{l_{n}}\right)^{2}\right)<\frac{e^{-\frac{m-1}{2}(t-1)^{2}}}{\sqrt{2 e(m-1)}(t-1)} \mathrm{E}\left(|u|^{2}\right)
$$

(we drop the standard calculation). It $I$ is a neighborhood of 1 , then the integrand in (5.14) decays exponentially outside $I$ as $m$ grows. Using arguments of Proposition 4.1, it is possible to prove that $1-\frac{\mathrm{E}\left(\left|\pi_{n} u\right|^{2}\right)}{\mathrm{E}\left(|u|^{2}\right)} \leq e^{-K m}$ for sufficiently large $n$, where $K$ depends only on $I$.

\section{References}

[1] Axler, S., Bourdon, P., Ramey, W., Harmonic function theory, 2nd ed., Graduate Texts in Mathematics. 137. New York, Springer, xi, $259 \mathrm{p}$

[2] Bateman H., Erdelyi A., Higher transcendental functions, V.1, McGraw-Hill, New York (1953).

[3] Bloch A., Polya G., On the Zeros of Certain Algebraic Equations. Proc. London Math. Soc. 33, 102-114, 1932.

[4] Bogomolny E., Bohigas O., Leboeuf P., Quantum chaotic dynamics and random polynomials, J. Statist. Phys. 85 (1996), no. 5-6, 639-679.

[5] Bürgisser P., Average Euler characteristic of random real algebraic varieties, C. R. Acad. Sci. Paris, Ser. I 345 (2007), 507-512.

[6] Edelman A., Kostlan E., How many zeros of a random polynomial are real? Bull. Amer. Math. Soc. 32 (1995), 1-37.

[7] Federer H., Geometric Measure Theory, Springer, 1969

[8] Fyodorov, Y., Lerario, A., and Lundberg E., On the number of connected components of random algebraic hypersurfaces, Geometry and Physics, 95 (2015), 1-20.

[9] Gichev V.M., Some remarks on spherical harmonics, St. Petersburg Math. J. 20 (2009), 553567. (Original publication: Algebra i Analiz, tom 20 (2008), nomer 4 (Russian).)

[10] Gichev V.M., Metric properties in the mean of polynomials on compact isotropy irreducible homogeneous spaces, Analysis and Math. Physics, 3 (2013), No. 2, 119-144.

[11] Kac M., On the average number of real roots of a random algebraic equation, Bull. Amer. Math. Soc. 49, (1943). 314-320.

[12] Kostlan E., On the distribution of roots of random polynomials. In The work of Smale in differential topology, From Topology to Computation: Proceedings of the Smalefest, pages 419431. Springer, 1993.

[13] Kostlan E., On the expected number of real roots of a system of random polynomial equations, Foundations of computational mathematics, Proceedings of Smalefest 2000, World Scientific Publishing 2002, pp. 149-188.

[14] Littlewood J.E., Offord A.C., On the number of real roots of a random algebraic equation, J. London Math. Soc. vol. 13 (1938) pp. 288-295.

[15] Littlewood J.E., Offord A.C., On the number of real roots of a random algebraic equation II, Proc. Cambridge Philos. Soc. 35 (1939), 133-148.

[16] Podkorytov S. S., The mean value of the Euler characteristic of an algebraic hypersurface, Algebra i Analiz, 11(5):185-193, 1999. English translation: St. Petersburg Math. J. 11(5) (2000), pp. 853-860.

[17] Shub M., Smale S., Complexity of Bezouts theorem II: volumes and probabilities. In F. Eyssette and A. Galligo, editors, Computational Algebraic Geometry, volume 109 of Progress in Mathematics, pages 267-285. Birkhauser, 1993.

[18] Stein E.M., Singular integrals and differentiability properties of functions, Princeton, 1970. 
Sobolev Institute of Mathematics, Omsk Branch, ul. Pevtsova, 13,, 644099, Omsk, Russia

E-mail address: gichev@ofim.oscsbras.ru 